\title{
Surface-chemistry technology for microfluidics
}

\author{
Winky L W Hau ${ }^{1}$, Dieter W Trau², Nikolaus J Sucher ${ }^{3,4}$, \\ Man Wong ${ }^{5}$ and Yitshak Zohar ${ }^{1}$ \\ ${ }^{1}$ Department of Mechanical Engineering, Hong Kong University of Science and Technology, \\ Clear Water Bay, Kowloon, Hong Kong \\ ${ }^{2}$ Bioengineering Graduate Program, Hong Kong University of Science and Technology, \\ Clear Water Bay, Kowloon, Hong Kong \\ ${ }^{3}$ Biotechnology Research Institute, Hong Kong University of Science and Technology, \\ Clear Water Bay, Kowloon, Hong Kong \\ ${ }^{4}$ Department of Biology, Hong Kong University of Science and Technology, \\ Clear Water Bay, Kowloon, Hong Kong \\ ${ }^{5}$ Department of Electrical and Electronic Engineering, Hong Kong University of Science and \\ Technology, Clear Water Bay, Kowloon, Hong Kong \\ E-mail: mezohar@ust.hk
}

Received 27 August 2002, in final form 18 December 2002

Published 17 January 2003

Online at stacks.iop.org/JMM/13/272

\begin{abstract}
A new technology to pattern surface charges, either negatively or positively, using a standard photolithography process is introduced. A positively charged poly(allylamine hydrochloride) (PAH) layer is coated onto a negatively charged silicon oxide surface by electrostatic self-assembly (ESA). Combined with photolithography in a lift-off-based process, several different surface charge patterns were successfully produced. Due to definition of the pattern by photolithography, no limitations in the pattern geometry exist. Any surface charge pattern can be created to enable fine control of fluid motion in microfluidic devices. Physical properties of this PAH layer were characterized. The generation of a bi-directional shear flow was demonstrated by using alternating longitudinal surface charge pattern with a single driving force, i.e. an externally applied electric field inside a microchannel.
\end{abstract}

\section{Introduction}

Microfluidic devices have recently received significant attention since many applications in biotechnology involve manipulation of small objects, e.g. DNA or protein molecules, in fluid media. These applications motivate the development of technologies for driving and controlling fluid motion on a microscale. Microchannels are the basic components of all microfluidic devices. To drive and control fluids inside a microchannel, especially liquids, a very high pressure gradient is required. Surface forces dominate at small dimension and, thus, the friction increases dramatically. Therefore, the study and application of surface-driven electro-osmotic flow (EOF) become important.

Typically, silicon oxide (or glass) surfaces are negatively charged at neutral $\mathrm{pH}$ due to deprotonated silanol groups $\left(\equiv \mathrm{Si}-\mathrm{O}^{-}\right)$. When these surfaces come in contact with a solution containing ions, positive ions will be attracted to the surface forming an immobile layer and a diffuse layer (thickness of the order of nanometers, e.g. $100 \mathrm{~nm}$ for a $10^{-5} \mathrm{M} \mathrm{NaCl}_{(\mathrm{aq})}$ solution) on the surface [1]. If an electric field is applied, the positive ion diffuse layer will move along the field. Consequently, the rest of the fluid is dragged by this diffuse layer via shear stress, resulting in bulk motion. This phenomenon is known as electro-osmotic flow [2]. Electrokinetic micro-pumps make use of this principle to drive fluid flows and can generate pressure up to $2 \mathrm{MPa}$ [3]. To drive EOF, the surface charge magnitude is important because it determines the fluid flow velocity. To control EOF, both the sign of the surface charge and its distribution on the surface are critical. The direction of EOF depends on both the direction of the applied electric field and the sign of surface charge. Therefore, surface charge patterning techniques are the key to control fluid motion on a microscale. In fact, this is another 
exclusive advantage of using EOF over pressure-driven flow because local surface charge pattern enables local control of fluid motion.

One of the promising applications of controlling fluid motion is to generate vortical flow for mixing purposes. Inplane vortical flow has been generated in a diffuser/nozzle configuration [4]; this approach still relies on both pressuredriven flow and EOF. EOF-based bi-directional shear flow and out-of-plane vortical flow were generated respectively with longitudinal and transverse surface charge patterns [5]. The surface patterning techniques were based on either a laminar flow of a coating solution over a substrate surface [6] or micro molding [7]. Others used polyelectrolytes to control the electro-osmotic flow direction and mobility in plastic microdevices [8]. However, these techniques are not convenient for batch fabrication.

In this work we describe a technique capable to produce any desired surface charge pattern on a flat silicon oxide or glass substrate. Since this process makes use of standard photolithography and simple surface treatment procedures, it should be scalable for mass production. Furthermore, an inspection technique for surface charge pattern confirmation is presented. Physical properties of the PAH coating including its thickness, hydrophobicity, zeta-potential and thermal stability were investigated. Experimental results were obtained by video capturing of particle motion showing bi-directional shear flow generated by a simple longitudinal pattern.

\section{Pretreatment for surface charge enhancement}

Materials based on glass or silicon oxide that are in contact with water/air for some time always carry some negative charges on their surface. When a piece of glass is brought into contact with an aqueous solution, alkali ions are extracted from the solution. Hydrogen ions from the water penetrate into the glassy network and replace alkali ions, which return back into the solution, as expressed by the following process:

$$
\equiv \mathrm{Si}-\mathrm{ONa}+\mathrm{H}_{2} \mathrm{O} \rightleftharpoons \equiv \mathrm{Si}-\mathrm{OH}+\mathrm{Na}^{+}+\mathrm{OH}^{-}
$$

Silanol groups $(-\mathrm{OH})$ are thus formed on the glass surface. At neutral $\mathrm{pH}$ of 7 , a substantial part of the silanol groups is deprotonized to form negative surface charges $\left(-\mathrm{O}^{-}\right)$. The density of these negative charges is usually small and not controllable. Therefore, a chemical treatment procedure was applied to achieve maximum charge density and ensure reproducible results. High density of negatively charged groups promotes the coating of PAH and creates a high potential difference in positively charged surface areas, thus, enhancing shear flows.

The chemical treatment process is further experimented on a borosilicate glass wafer, $100 \mathrm{~mm}$ in diameter and $500 \mu \mathrm{m}$ in thickness (Corning, USA). The glass wafer was first cleaned in sulfuric acid $\left(\mathrm{H}_{2} \mathrm{SO}_{4}: \mathrm{H}_{2} \mathrm{O}_{2}=10: 1\right)$ at $120{ }^{\circ} \mathrm{C}$ for $10 \mathrm{~min}$ to remove organic impurities. After rinsing with copious amounts of deionized (DI) water, the wafer was submerged in $1 \%$ (wt) $\mathrm{NaOH}_{(\mathrm{aq})}$ solution at $90^{\circ} \mathrm{C}$ for $30 \mathrm{~min}$. Each hydroxide ion hydrolyzes the silicon oxide at the surface (figure 1(a)) to form one silanol group and one silanol salt group (figure 1(b)) as follows:

$$
\equiv \mathrm{Si}-\mathrm{O}-\mathrm{Si} \equiv+\mathrm{Na}^{+}+\mathrm{OH}^{-} \rightleftharpoons \equiv \mathrm{Si}-\mathrm{OH}+\equiv \mathrm{Si}-\mathrm{ONa}
$$

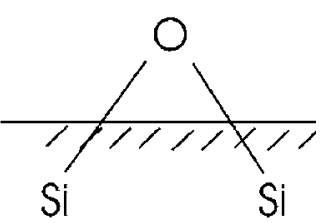

(a)

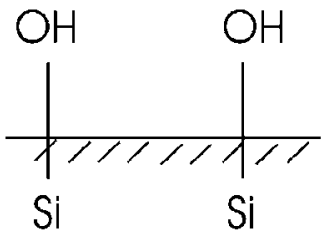

(c)

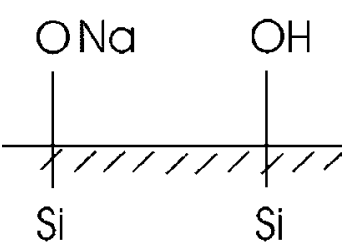

(b)

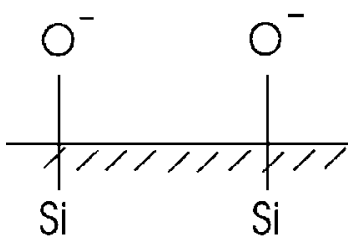

(d)
Figure 1. Treatment of glass surface: $(a)$ untreated silica surface, (b) after treatment with $\mathrm{NaOH}_{(\mathrm{aq})}$, (c) after treatment with $\mathrm{HCl}_{(\mathrm{aq})}$ and $(d)$ after interaction with water at $\mathrm{pH} 7-8$.

After washing away the residual $\mathrm{NaOH}_{(\mathrm{aq})}$ from the surface, the wafer was submerged in $3 \%$ (vol.) $\mathrm{HCl}_{(\mathrm{aq})}$ solution at room temperature for $10 \mathrm{~min}$. The hydrogen ions in hydrochloric acid displace the sodium ions and convert all groups into silanol groups (figure $1(c)$ ):

$$
\equiv \mathrm{Si}-\mathrm{ONa}+\mathrm{H}^{+}+\mathrm{Cl}^{-} \rightleftharpoons \equiv \mathrm{Si}-\mathrm{OH}+\mathrm{Na}^{+}+\mathrm{Cl}^{-}
$$

Finally, the wafer was washed again with copious amounts of DI water and preferably stored under DI water. The silanol groups are deprotonized at neutral $\mathrm{pH} 7$ resulting in a negatively charged surface (figure $1(d)$ ):

$$
\equiv \mathrm{Si}-\mathrm{OH} \rightleftharpoons \equiv \mathrm{Si}-\mathrm{O}^{-}+\mathrm{H}^{+}
$$

\section{Electrostatic self-assembly method}

Positively charged surfaces were produced by electrostatic self-assembly (ESA) of a positively charged polyelectrolyte. A polyelectrolyte is a polymeric molecule that carries a high number of electrically charged functional groups. In our study, poly(allylamine hydrochloride) $(\mathrm{PAH})$ with positively charged amino groups (at $\mathrm{pH}$ 6-7) was used.

Prior to use, the PAH was covalently conjugated with fluorescein isothiocyanate (FITC). FITC is a fluorescent molecule and will later be used to visualize PAH-coated areas.

The PAH-FITC conjugate was synthesized as follows: $500 \mathrm{mg}$ PAH (15000 $\mathrm{g} \mathrm{mol}^{-1}$, Aldrich, USA) was dissolved in $6 \mathrm{ml}$ DI water and the $\mathrm{pH}$ was adjusted to $\mathrm{pH} 8.0-8.5$ with $1 \mathrm{M} \mathrm{NaOH}_{(\mathrm{aq})}$ solution. A second solution of $4 \mathrm{mg}$ FITC (Isomer I, 90\% purity, Aldrich, USA) in $500 \mu \mathrm{l}$ dimethylsulfoxide (DMSO) (Sigma, USA) was prepared. The FITC solution was mixed with the PAH solution and incubated in the dark (to prevent bleaching of FITC) over night at room temperature. The conjugation ratio FITC:PAH-monomer was 1:500. The PAH-FITC reaction mixture was filled into a Slide-A-Lizer cassette (2000 Dalton exclusion size, Pierce, USA) and dialyzed against DI water overnight to remove nonconjugated FITC molecules. No change in color of the DI 

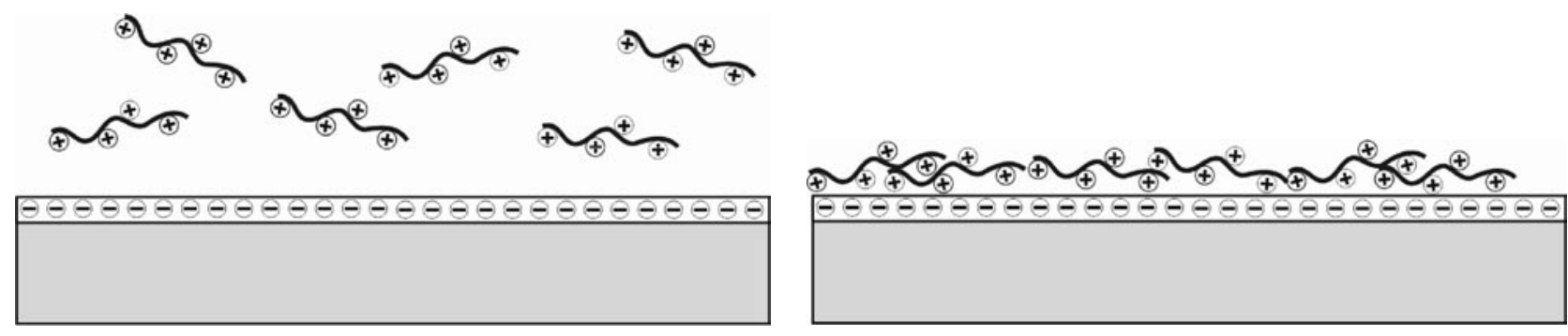

(a)

(b)

Figure 2. Electrostatic self-assembly process: $(a)$ the substrate is submerged in PAH solution, and $(b)$ PAH is coated onto the silicon oxide surface due to electrostatic attraction.

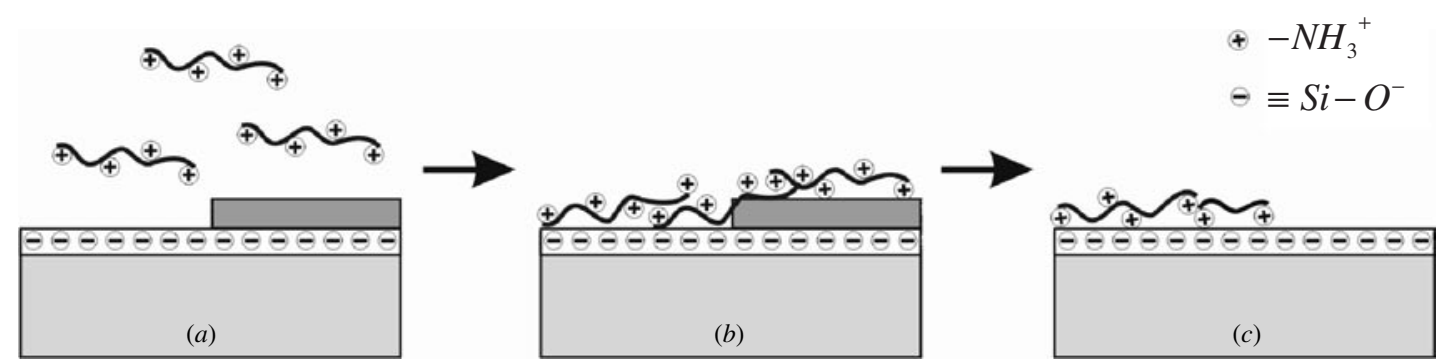

Figure 3. Lift-off surface charge patterning technique: $(a)$ silicon oxide surface with patterned photoresist, $(b)$ electrostatic self-assembly process for PAH and (c) photoresist removal.

water used to dialyze the conjugate was observed, indicating that almost all FITC molecules were conjugated to PAH. Finally, the PAH-FITC solution was adjusted to $\mathrm{pH} 6$ with $1 \mathrm{M} \mathrm{HCl}_{(\mathrm{aq})}$. At this $\mathrm{pH}$, the amino functionality of the PAH is protonated and positively charged. The conjugate was stored at $4{ }^{\circ} \mathrm{C}$ in the dark. A coating solution of $9 \mathrm{mg} \mathrm{ml}^{-1} \mathrm{PAH}-$ FITC was prepared from the stock solution by dilution with water prior to usage.

The principle of the ESA process is illustrated in figure 2. A glass wafer with a $\mathrm{NaOH}_{(\mathrm{aq})}$ and $\mathrm{HCl}_{(\mathrm{aq})}$ treated surface was submerged into the PAH-FITC coating solution for $30 \mathrm{~min}$. PAH-FITC was deposited onto the glass surface driven by electrostatic attraction (coulomb interaction) between the negatively charged glass surface and the positively charged PAH molecule. Finally, the glass wafer was washed with DI water.

\section{Surface charge patterning technique}

The surface charge patterning technique is based on standard photolithography followed by ESA of PAH and removal of the photoresist (PR) coated with the PAH in a lift-off process.

The starting material is a $\mathrm{NaOH}_{(\mathrm{aq})}$ and $\mathrm{HCl}_{(\mathrm{aq})}$ treated glass wafer with enhanced negative surface charge. A PR film (PR204, Fujifilm Olin Co. Ltd, Japan) was spin-coated (4000 rpm, $30 \mathrm{~s}$ ) onto the glass surface. A mask was aligned with the wafer, and the PR was patterned and developed by standard photolithography. The wafer was immersed into PAH-FITC solution (figure 3(a)) for $30 \mathrm{~min}$ allowing the deposition of PAH-FITC onto the surface (figure 3(b)). After washing with water, the wafer was incubated in acetone for 10 min to remove the PR and the PAH-FITC on top of it in a lift-off process (figure 3(c)). Finally, the glass wafer was washed with ethanol and DI water and dried with nitrogen gas. In this technique, a pattern of positively charged $\mathrm{PAH}$ and negatively charged glass surface was produced.

\section{Inspection technique}

After the surface charge patterning process was completed, the success of the procedure was verified by an inspection technique based on fluorescent microscopy. The existence of the PAH coating as well as the coating uniformity and the quality of the edges $\mathrm{PAH} /$ glass can be investigated by the inspection.

A light micrograph of a PAH pattern on a silicon wafer having a silicon oxide surface is shown in figure $4(a)$. The wafer was illuminated from the top with white light. The pattern is clearly seen on the nontransparent silicon wafer. However, it was difficult to verify the pattern on transparent glass wafers. More information can be obtained by using fluorescent microscopy. Only areas coated with FITC labeled PAH will emit light with intensity proportional to the amount of PAH coating material when excited, while uncoated areas will remain dark. Figure $4(b)$ shows an image, of the same sample shown in figure 4(a), obtained by fluorescence confocal scanning (ScanArray 5000, Packard Bioscience, USA). Fluorescent light originated from the square-like areas confirms the presence of the desired PAH-FITC pattern on the surface. Furthermore, the image confirms that the PAHFITC survived the treatment in acetone and ethanol for the PR removal. The uniform light intensity originated from the PAH area indicates uniform thickness and charge density. In addition, the totally dark silicon oxide area and the sharp boundary between the various areas confirm the success of the technique. 


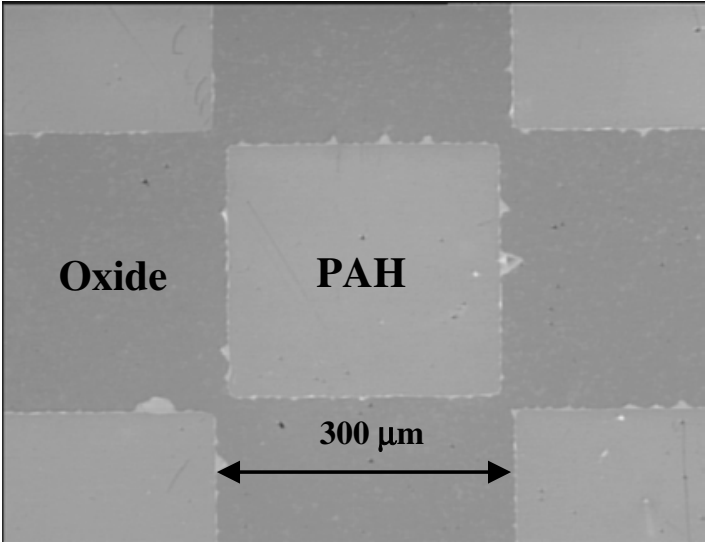

(a)

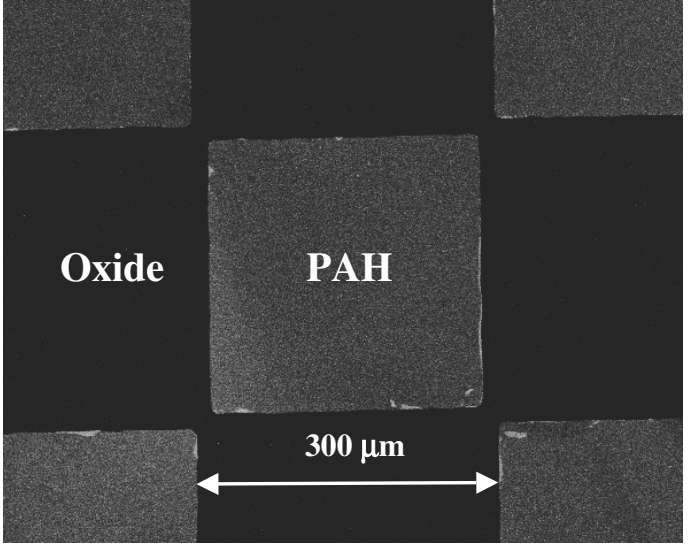

(b)

Figure 4. PAH pattern after surface charge patterning: (a) microscopic image and $(b)$ confocal fluorescent microscopic image.

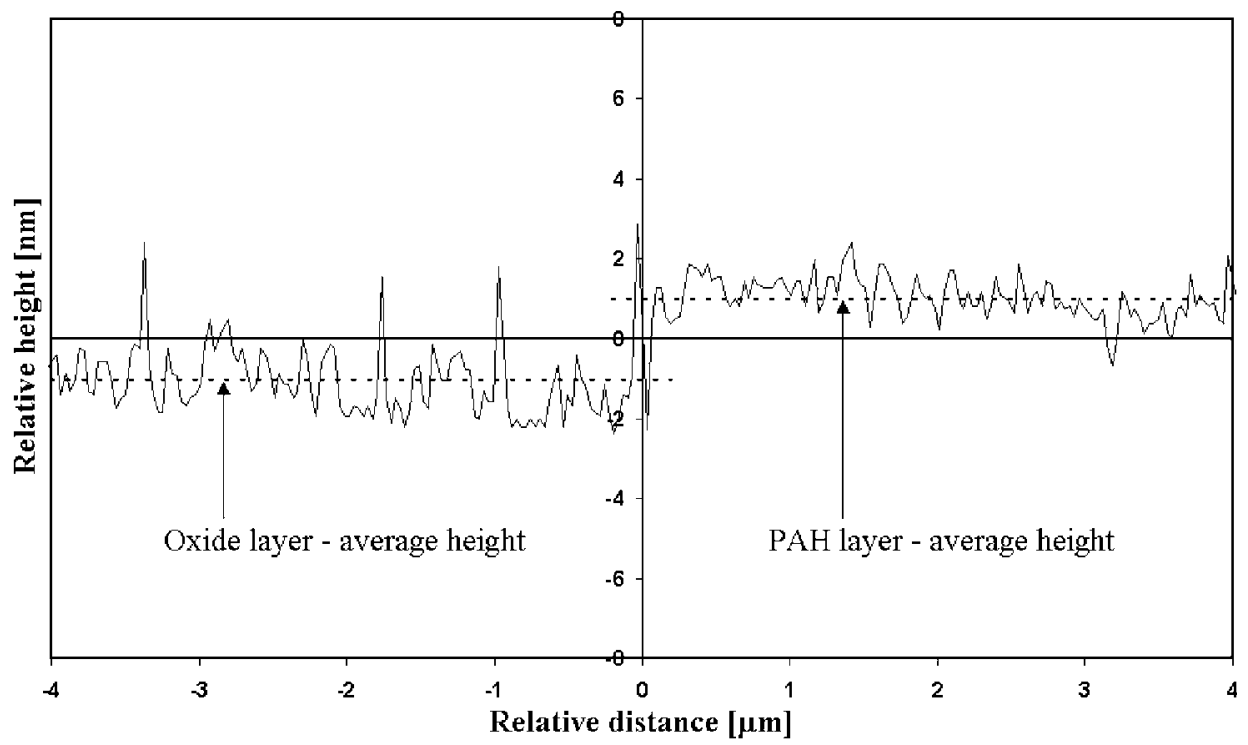

Figure 5. AFM profile scan across the boundary between oxide and PAH region; the height of the PAH coating is about $2 \mathrm{~nm}$.

\section{Physical properties}

For application in EOF-based microdevices, the following physical properties of surface coatings are desired: the thickness of the surface coating should be small in comparison to the channel dimensions. The surface charge should be high to generate a strong EOF. The stability of the coating should be high and constant. Surface hydrophobicity will affect capillary forces in microchanels and can influence the loading characteristics dramatically. However, the desired hydrophobicity depends on the application.

Thickness. The thickness and surface morphology of the PAH coating was studied by atomic force microscopy (AFM) (Nanoscope, Digital Instruments, USA). The dry sample was inspected immediately after fabrication to avoid any potential swelling of the coated film, and the surface profile is shown in figure 5. In fact, the thickness of polyelectrolyte coating was reported to depend on several factors such as molar mass, $\mathrm{pH}$ value, ionic strength of the solution and the substrate surface. The measured thickness of the PAH coating in the present work, about $2 \mathrm{~nm}$, is consistent with measurements reported by others [9-11]. This thickness is about three orders of magnitude smaller than channel height in typical microdevices and, therefore, will not affect the channel cross section.

Surface charge. The surface charge expressed as the zetapotential determines the bulk fluid velocity of EOF. The zetapotential of PAH and silicon oxide can be calculated by the following equation:

$$
u=-\frac{E \varepsilon \varepsilon_{0} \zeta}{\mu}
$$

where $\zeta$ is the zeta-potential, $u$ is the bulk fluid velocity, $E$ is the applied electric field, $\varepsilon$ is the dielectric constant of water, $\varepsilon_{0}$ is the absolute permittivity and $\mu$ is the dynamic viscosity. When an electric field $E$ was externally applied in parallel to the surface, the liquid was induced into motion with velocity $u$. Hence, the zeta-potential $\zeta$ of the surface can be estimated from the measured velocity $u$. Video capturing of moving individual microparticles (PS, micro particles $\mathrm{GmbH}$, Germany) was used to measure bulk flow velocity at a given area and study micro-flow patterns as illustrated in figure 6 . 


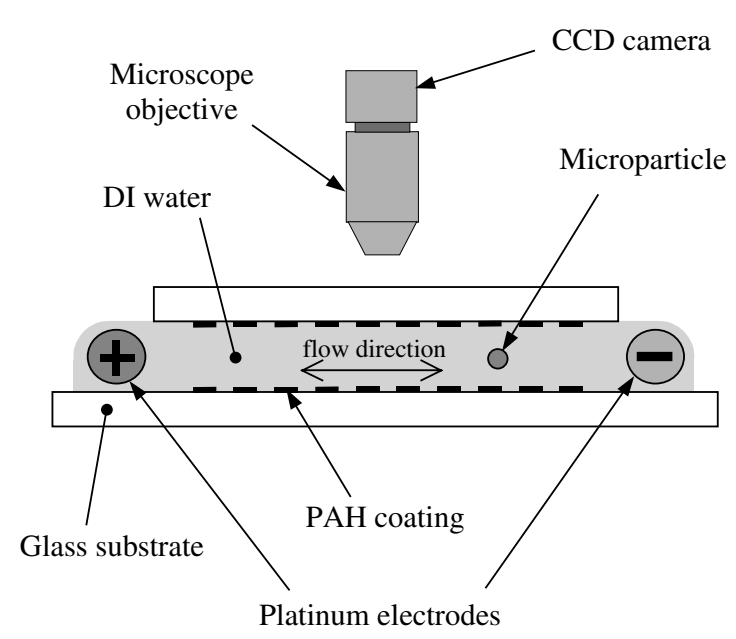

Figure 6. A schematic diagram illustrating the experimental set-up used for the particle velocity measurement.

A microparticle velocity of $150 \mu \mathrm{m} \mathrm{s}^{-1}$ was measured for both the PAH surface and silicon oxide surfaces. The velocities are of the same magnitude but in opposite direction (figure 7). From equation (1), the zeta-potentials of the PAH surface and silicon oxide surface were calculated as $+65 \mathrm{mV}$ and $-65 \mathrm{mV}$, respectively. These results should be considered as rough estimates only. The electrophoretic force on the negatively charged particles is expected to increase the measured particle velocity over the PAH surface and decrease the velocity over the oxide surface. An attempt was made to quantify this effect by measuring the particle velocity under similar electric field over a neutral surface. Since no detectable motion was observed, the electrophoretic effect was neglected.

Stability. The stability of the coating is a concern in the following cases: during the device fabrication, the coating must possibly withstand a high temperature ( 80 to $200{ }^{\circ} \mathrm{C}$ ) for a short period of time $(<1 \mathrm{~h})$. If the device is stored, the dry coating should have good long-term stability (1 year) at room temperature $\left(<30{ }^{\circ} \mathrm{C}\right)$. If the device is used, the coating should be stable while in contact with a liquid at moderate temperature $\left(20\right.$ to $40{ }^{\circ} \mathrm{C}$ ) for several hours. It was reported that a dry PAH coating could be sustained at $200{ }^{\circ} \mathrm{C}$ for 1 week [12]. Thermal stability can be further enhanced by cross linking. Our preliminary results indicate that the film can be stored for more than 4 months and still exhibits EOF characteristic. Repeated application and cleaning cycles with water and ultrasound were carried out with the same surface pattern, indicating a robust coating (non-oxidative aquatic solvent, temperature below $40^{\circ} \mathrm{C}$ ).

Hydrophobicity. The surface hydrophobicity of PAH and silicon oxide was found to be different. When water vapor condensed onto the wafer surface, a difference in droplet size was observed. This phenomenon is demonstrated in figure 8 on an alternating longitudinal pattern of PAH and silicon oxide stripes (each stripe is $100 \mu \mathrm{m}$ in width). The droplets on the silicon oxide surface are on average much bigger than those on the PAH surface due to differences in hydrophobicity. Contact angles of $49^{\circ}$ for the PAH surface (figure $9(a)$ ) and $18^{\circ}$ for the silicon oxide surface (figure $9(b)$ ) were measured (Krüss Contact Angle Measuring System G10). Evidently, the PAH surface is more hydrophobic than the silicon oxide surface.

\section{Flow pattern example}

A longitudinal pattern was used to demonstrate the potential of the described surface charge patterning technique for fluid control. A microchannel of $50 \mu \mathrm{m}$ height was formed by bonding two patterned glass substrates and using double-sided adhesive tape. The surface pattern was made of alternating longitudinal stripes, $100 \mu \mathrm{m}$ in width, carrying positive and negative charges. The channel was filled with DI water, and a potential was applied between platinum electrodes at both ends of the channel. Microparticles of $1.8 \mu \mathrm{m}$ diameter were injected into the microchannel for flow visualization. Figure 10 shows a light microscopic image of the generated shear flow pattern. It is clearly seen that every second stripe contains higher microparticle density. This phenomenon is due to the fact that the microparticles carry negative charges and, hence, were attracted to the positively charged PAH surface. The high concentration in the positive areas implies that the
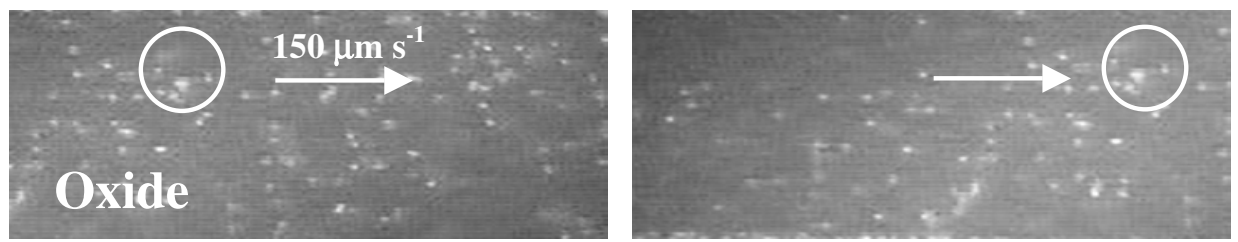

(a)
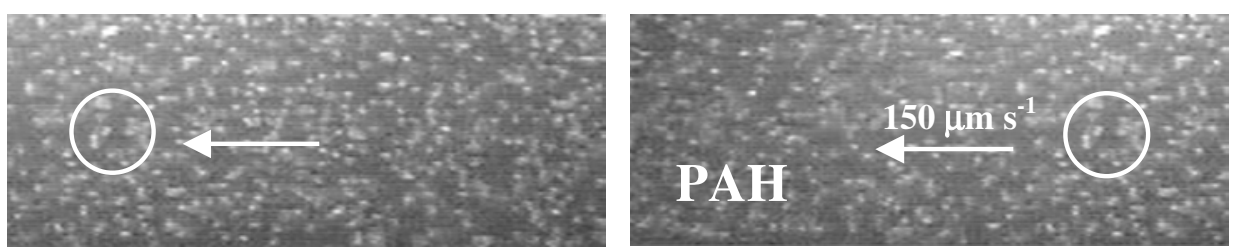

(b)

Figure 7. Velocity measurement of EOF-driven microparticles over ( $a$ ) oxide surface (left-to-right) and $(b)$ PAH surface (right-to-left). 


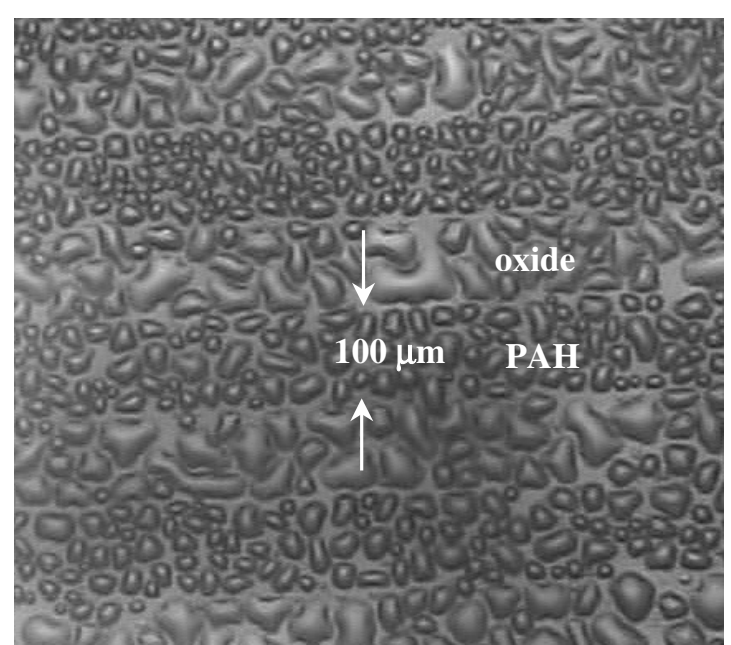

Figure 8. Condensed water droplets demonstrate hydrophobicity difference between oxide surface and PAH surface.

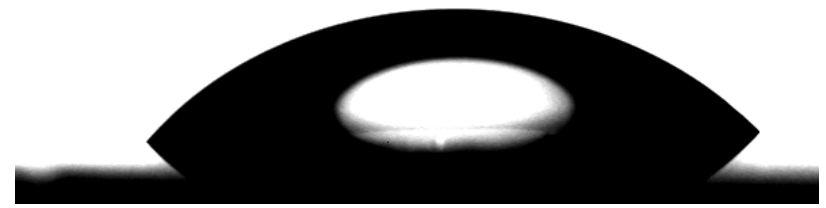

(a)

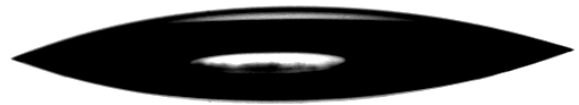

(b)

Figure 9. Contact angle measurements over (a) PAH surface, $49^{\circ}$, and $(b)$ oxide surface, $18^{\circ}$.

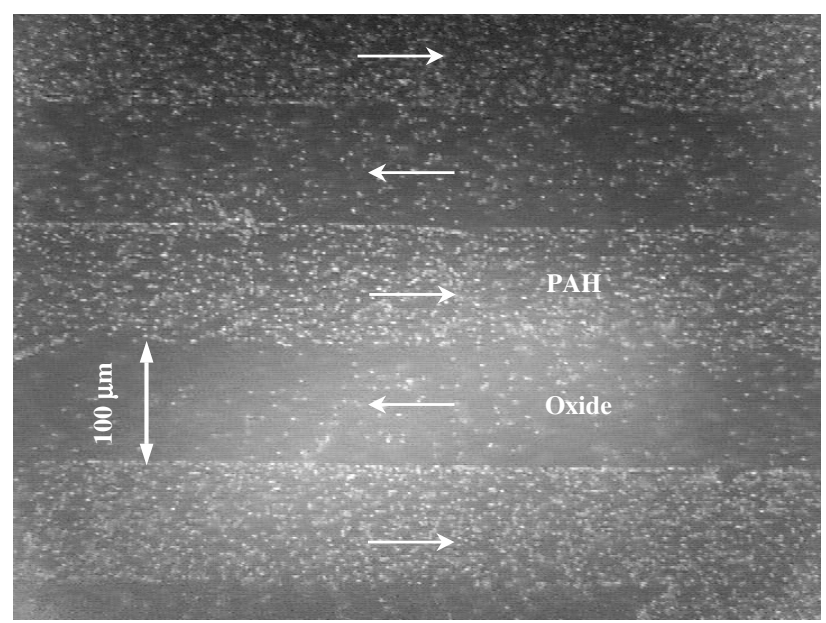

Figure 10. Shear flow on a longitudinal-pattern surface charge.

particles are interacting with the surface. This would decrease the apparent mobility of the particles, opposite the enhanced mobility due to the electrophoretic force. Indeed, the motion of the microparticles over PAH surface and silicon oxide surface was observed to be in opposite direction that is consistent with the prediction based on equation (1).

\section{Conclusions}

A simple technique for zeta-potential patterning of flat silicon oxide or glass surfaces with electrostatic self-assembled poly(allylamine hydrochloride) is introduced. The merit of the technique is that it is based on standard photolithography. Therefore, any desired geometry of positive and negative surface charge patterns can be produced. This greatly facilitates the design and control of micro-flow patterns. The positively charged PAH surface coating has a thickness of about $2 \mathrm{~nm}$ and shows long-term stability. The generation of bi-directional shear flow patterns was demonstrated proving the feasibility of the approach. Flow velocity and zeta-potentials were measured in an experiment that uses microparticles as a probe. The velocity of the microparticles within each strip was observed to be uniform. This indicates that the microparticle motion is directly controlled by the underneath surface charge only and, hence, liquid flow control is possible on a microscale. Currently we are focusing on the generation of various other micro-flow patterns, e.g. vortical flow, for mixing purposes and their applications in BioMEMS.

\section{Acknowledgments}

This work is supported by the Hong Kong Research Grant Council through RGC grant HKUST6082/00E, the Industry Department of the Hong Kong SAR (AF/150/99), and the Hong Kong Jockey Club.

\section{References}

[1] Hunter R J 1981 Zeta Potential in Colloid Science: Principles and Applications (London: Academic)

[2] Grossman P D and Colburn J C 1992 Capillary Electrophoresis: Theory and Practice (San Diego: Academic)

[3] Zeng S, Chen C-H, Mikkelsen J and Santiago J G 2000 Fabrication and characterization of electrokinetic micro pumps 7 th Intersociety Conf. on Thermal and Thermomechanical Phenomena in Electronic Systems vol 2 pp 31-6

[4] Lettieri G, Verpoorte E and de Rooij N 2001 Planar micro-fluidic devices for controlled vortex generation 11th Int. Conf. Solid-State Sensors Actuators vol 2 pp 1510-3

[5] Stroock A D, Weck M, Chiu D T, Huck W T C, Kenis P J A, Ismagilov R F and Whitesides G M 2000 Patterning electro-osmotic flow with patterned surface charge Phys. Rev. Lett. 84 3314-7

[6] Kenis P J A, Ismagilov R F and Whitesides G M 1999 Microfabrication inside capillaries using multiphase laminar flow patterning Science 285 83-85

[7] Jeon N L, Choi I S, Xu B and Whitesides G M 1999 Large-area patterning by vacuum-assisted micromolding Adv. Mater. 11 946-50

[8] Barker S L R, Tarlov M J, Canavan H, Hickman J J and Locascio L E 2000 Plastic microfluidic devices modified with polyelectrolyte multilayers Anal. Chem. 72 4899-902 
[9] Lvov Y, Onda M, Ariga K and Kunitake T 1988 Ultrathin films of charged polysaccharides assembled alternately with linear polyions J. Biomater. Sci. Polym. Ed. $9345-55$

[10] Hoogeveen N G, Cohen Stuart M A and Fleer G J 1996

Polyelectrolyte adsorption on oxides: I. Kinetics and adsorbed amounts J. Colloid Interface Sci. 182 133-45
[11] Yoo D, Shiratori S S and Rubner M F 1998 Controlling bilayer composition and surface wettability of sequentially adsorbed multilayers of weak polyelectrolytes Macromolecule 31 4309-18

[12] Arys A, Jonas A M, Laschewsky A and Legras R 2000 Supramolecular Polymers (New York: Dekker) 